\title{
Single Particle X-ray Diffractive Imaging
}

M. J. Bogan, W. H. Benner, S. Boutet, U. Rohner, M. Frank, M. Seibert, F. Maia, A. Barty, S. Bajt, V. Riot, B. Woods, S. Marchesini, S. P. Hau-Riege, M. Svenda, E. Marklund, E. Spiller, J. Hajdu, H. N. Chapman

October 9, 2007

Nano Letters 
This document was prepared as an account of work sponsored by an agency of the United States government. Neither the United States government nor Lawrence Livermore National Security, LLC, nor any of their employees makes any warranty, expressed or implied, or assumes any legal liability or responsibility for the accuracy, completeness, or usefulness of any information, apparatus, product, or process disclosed, or represents that its use would not infringe privately owned rights. Reference herein to any specific commercial product, process, or service by trade name, trademark, manufacturer, or otherwise does not necessarily constitute or imply its endorsement, recommendation, or favoring by the United States government or Lawrence Livermore National Security, LLC. The views and opinions of authors expressed herein do not necessarily state or reflect those of the United States government or Lawrence Livermore National Security, LLC, and shall not be used for advertising or product endorsement purposes. 
2 Michael J. Bogan, ${ }^{1 *}$ W. Henry Benner, ${ }^{1}$ Sébastien Boutet, ${ }^{1,2,3}$ Urs Rohner, ${ }^{1}$ Matthias 3 Frank, ${ }^{1}$ Anton Barty, ${ }^{1}$ Marvin Seibert, ${ }^{3}$ Filipe Maia, ${ }^{3}$ Stefano Marchesini, ${ }^{1 \dagger}$ Saša Bajt, ${ }^{1 \dagger \dagger}$ 4 Bruce Woods, ${ }^{1}$ Vincent Riot ${ }^{1}$, Stefan P. Hau-Riege, ${ }^{1}$ Martin Svenda, ${ }^{3}$ Erik Marklund, ${ }^{3}$ 5 Eberhard Spiller, ${ }^{4}$ Janos Hajdu, ${ }^{2,3}$ and Henry N. Chapman ${ }^{1,5}$

6 1. Lawrence Livermore National Laboratory, 7000 East Avenue, Livermore CA 94550, USA.

2. Stanford Synchrotron Radiation Laboratory, Stanford Linear Accelerator Center, 2575 Sand Hill Road, Menlo Park, California 94305, USA.

3. Laboratory of Molecular Biophysics, Institute of Cell and Molecular Biology, Uppsala University, Husargatan 3, Box 596, S-75124 Uppsala, Sweden.

5. Centre for Free-Electron Laser Science, Universität Hamburg, Notkestraße 85, 22607 Hamburg, Germany.

15 Author email address: bogan2@1ln1.gov

16 *Corresponding author: Michael J. Bogan, Applied Physics and Biophysics, Lawrence 17 Livermore National Laboratory, 7000 East Avenue L-211, Livermore CA 94550, USA

18 Current Addresses: $\uparrow$ Advanced Light Source, MS 2-400, Lawrence Berkeley National

19 Laboratory, 1 Cyclotron Road, Berkeley, CA 94720, USA; $\dagger \dagger$ Deutches Elektronen

20 Synchrotron, Notkestraße 85, 22607 Hamburg, Germany.

21 Table of Contents Figure:

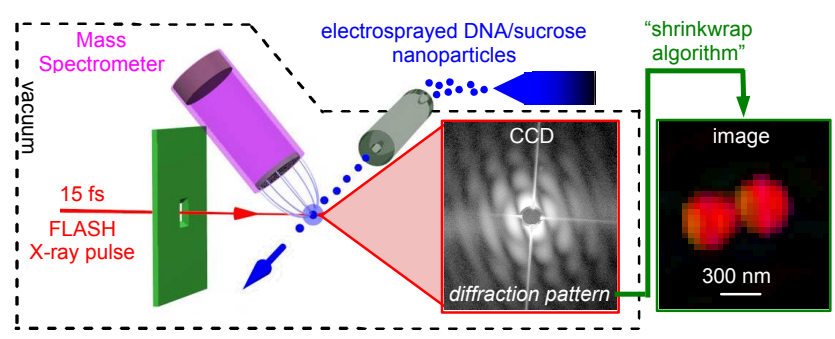


1 ABSTRACT: In nanotechnology, strategies for the creation and manipulation of 2 nanoparticles in the gas phase are critically important for surface modification and 3 substrate-free characterization. Recent coherent diffractive imaging with intense 4 femtosecond $\mathrm{X}$-ray pulses has verified the capability of single-shot imaging of 5 nanoscale objects at sub-optical resolutions beyond the radiation-induced damage 6 threshold. By intercepting electrospray-generated particles with a single 15 7 femtosecond soft-X-ray pulse, we demonstrate diffractive imaging of a nanoscale 8 specimen in free flight for the first time, an important step toward imaging uncrystallized biomolecules.

10 KEYWORDS: free-electron laser, electrospray, aerodynamic lens, single particle, X-ray 11 diffractive imaging, DNA, nanoparticles

12 Development of methods enabling higher spatial and time resolution characterization of 13 nanoscale objects such as single particles and biomolecules is essential to achieve the 14 goals of nanotechnology and to push structural biology into new frontiers. We are 15 developing strategies to enable characterization of nanoscale objects using the first 16 operational soft X-ray free electron laser (FEL), the FLASH facility at DESY in 17 Hamburg, Germany. ${ }^{1}{ }^{2}$ This facility produces ultrafast $(<30 \mathrm{fs})$ and ultrabright coherent 18 X-ray pulses (peak brightness $>10^{30}$ photons $/ \mathrm{s} / \mathrm{mrad}^{2} / \mathrm{mm}^{2} /\left(0.1 \%\right.$ bandwith), or $>10^{12}$ 19 photons/pulse) and provides many new possibilities for probing nanoscale objects. ${ }^{3}$ 20 Examples of new capabilities demonstrated at FLASH include femtosecond time21 resolved holography for measurements of nanoparticle dynamics ${ }^{4}$ and single-pulse 22 diffractive imaging of nanostructured materials. ${ }^{5}$ In diffractive imaging a sample is 23 illuminated by coherent X-rays, a far-field diffraction pattern is recorded on an area 24 detector, such as a CCD, and the image is reconstructed using iterative phase retrieval 25 algorithms. 6, 7 During initial experiments at FLASH, samples were mounted on 26 supporting silicon nitride membranes that contributed background scattering to the 27 measurements. When the number of atoms in the substrate becomes much larger than the 28 number of atoms in the sample itself, such as in the case of isolated biomolecules on a 29 membrane, this background is expected to dominate the signal measured by the CCD. 30 This motivates the development of sample handling systems free of supporting 
1 membranes, such as particle beams, for introducing particles and biomolecules into the

2 X-ray beam. Successful implementation of such container-free sampling methods may

3 enable structural determinations without the need for a crystal. ${ }^{8-10}$

5 Particle beams have been at the vanguard of research in most branches of science.

6 Recently, strategies for manipulating nanoparticles in the gas phase using their inertial

7 properties are gaining interest in the manufacture of nanostructured materials ${ }^{11,12}$ and for

8 substrate-free particle characterization in vacuo. ${ }^{13-15}$ A key development in the generation

9 of focused nanoparticle beams in vacuo was Liu et al.'s implementation of thin plate

10 orifices to manipulate particle spatial distribution prior to them passing through a nozzle

11 and subsequently undergoing supersonic expansion. ${ }^{16,17} \mathrm{An}$ axisymmetric stack of these

12 thin plate orifices, or aerodynamic lenses, provides successive contractions of a flowing

13 particle beam and enables focusing of a wide range of particle sizes ( $1 \mathrm{~nm}$ to $10 \mu \mathrm{m}){ }^{18} \mathrm{In}$

14 this letter we demonstrate a viable technique, based on such an aerodynamic lens stack,

15 for introducing isolated single particles in free flight into a pulsed X-ray beam for

16 substrate-free imaging. We demonstrate an imaging resolution of better than $40 \mathrm{~nm}$ by

17 diffractive imaging with the single X-ray pulse, and further characterize the interaction

18 by mass spectrometry.

20 Substrate-Free Particle Delivery to X-rays. Our experimental geometry (Figure 1) is 21 based on that previously used to image samples on silicon nitride foils. ${ }^{5}$ Pulses from the

22 FLASH FEL are condensed to a $20-\mu \mathrm{m}$ diameter beam at the sample plane using a 23 grazing-incidence ellipsoidal mirror. No monochromator or pinhole is used to limit the

24 beam since the FEL output has sufficient temporal and spatial coherence for diffractive 25 imaging. Instead of micro-positioning a substrate-supported sample into the beam, the 26 apparatus is designed to introduce a continuous particle beam transmitted by an 27 aerodynamic lens stack into the FEL beam. To steer the particle beam to intersect with 28 the X-ray beam, the aerodynamic lens stack is mechanically translated. This is achieved 29 by translating the entire differential pumping interface, represented in Fig. 1 (iv), in 30 which the lens stack is integrated 
1 The sample is generated using a charge-reduced nanoelectrospray aerosol source. ${ }^{19}$ The

2 method is well characterized, has previously been used to prepare samples of spherical 3 nanoparticles on silicon nitride foils for characterization at $\mathrm{FLASH},{ }^{20}$ and is applicable to

4 a wide variety of nanoscale materials that are likely to be characterized using FELs 5 including carbon nanotubes, ${ }^{21}$ large biomolecules, ${ }^{22}$ viruses ${ }^{23-25}$ and cells. ${ }^{26}$ One 6 particular sample of interest is scaffolded DNA origami, consisting of double-stranded 7 DNA programmed to form predefined two-dimensional geometrical shapes. ${ }^{27} \mathrm{We}$ are 8 investigating DNA nanostructures because they may be useful as nano-breadboards for 9 the arrangement of nano-objects or biomolecules for enhancing the signal-to-noise ratio 10 in single particle imaging methods, including holography with a complicated reference. ${ }^{28}$
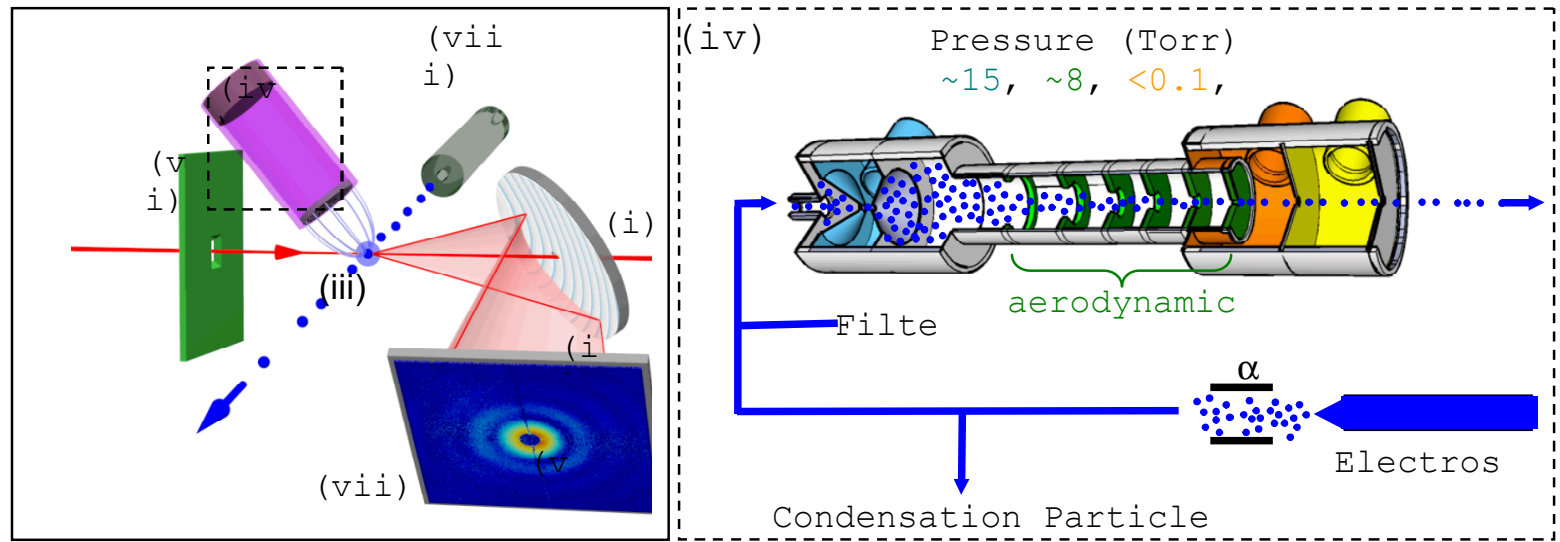

11 Figure 1. Schematic diagram of the experimental apparatus (not to scale). The FEL pulses (i) are incident from the right, pass through an aperture designed to block stray 13 light (ii) and are focused to a $20 \mu \mathrm{m}$ spot that defines the interaction region (iii). 14 Electrospray-generated aerosol is charge-neutralized via an $\alpha$ source and delivered into vacuum through a differential pumping interface equipped with an aerodynamic focusing system (iv). The pressure in the three pumping regions, coloured blue, orange and yellow, are set to optimize the focusing of the nanoparticles through an aerodynamic lens stack 18 (green). The resultant continuous stream of nanoparticles travels at $\sim 150 \mathrm{~m} / \mathrm{s}$ and is confined to a beam of $\sim 250-\mu \mathrm{m}$ diameter (v). The nanoparticles are steered into the interaction region using mechanical translation of the entire differential pumping interface. X-rays diffracted from the interception of single nanoparticles by the FEL reflect from a graded multilayer planar mirror (vi) to the single photon sensitive X-ray $\mathrm{CCD}$ (vii) while the direct beam passes through a hole in the centre of the mirror. Ions created from the FEL interactions are detected by a miniature time-of-flight mass 25 spectrometer (viii). 
1 For the first experiments it was essential to use particles that would produce strong

2 diffraction patterns. When electrosprayed, individual megadalton DNA complexes have

3 an aerodynamic diameter of $28 \mathrm{~nm}$ (Figure 2). The X-ray intensity produced by focusing

4 the beam to a diameter of $20-\mu \mathrm{m}$ was not sufficient to record single pulse diffraction

5 patterns of these particles. We increased the signal by enlarging the particles to increase

6 their scattering cross section. Larger particles were created using Kaufman's in-situ

7 electrospray method for synthesis of sucrose encapsulated biomolecules. ${ }^{29}$ Sucrose is

8 added to the starting solution and upon evaporation of the dilute ammonium acetate/water

9 buffer, sucrose-encapsulated DNA is created. The diameter of the composite nanoparticle

10 is given by $\left[\mathrm{d}_{\mathrm{m}}{ }^{3}+\mathrm{d}_{\text {suc }}{ }^{3}\right]^{1 / 3}$, where $\mathrm{d}_{\mathrm{m}}$ and $\mathrm{d}_{\text {suc }}$ are the diameters of the discrete

11 macromolecule (28 $\mathrm{nm}$ for the DNA complexes) and residue particles of sucrose ( $80 \mathrm{~nm}$ )

12 when electrosprayed separately. ${ }^{29}$ The composite sucrose/DNA particles averaged $81 \mathrm{~nm}$

13 in diameter and their mass is dominated by the sucrose (Figure 2). Particles larger or

14 smaller than $81 \mathrm{~nm}$ resulted from variations in the initial volume of droplets emitted and

15 from periodic instability of the electrospray source. All particle sizes are directed toward 16 the FEL.

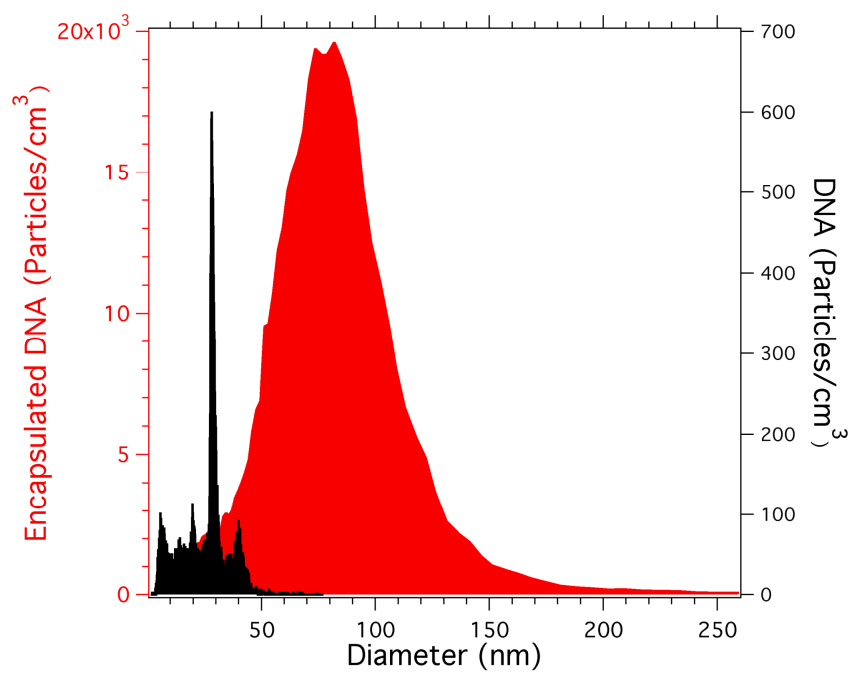

17 Figure 2. Size distribution of scaffolded DNA electrosprayed from a $25 \mathrm{mM}$ 18 ammonium acetate solution containing $0 \%$ (black) and $50 \%$ (red) sucrose, as measured 19 by scanning mobility particle size spectrometry. Both bare DNA and sucrose20 encapsulated DNA were injected into the FEL beam. 


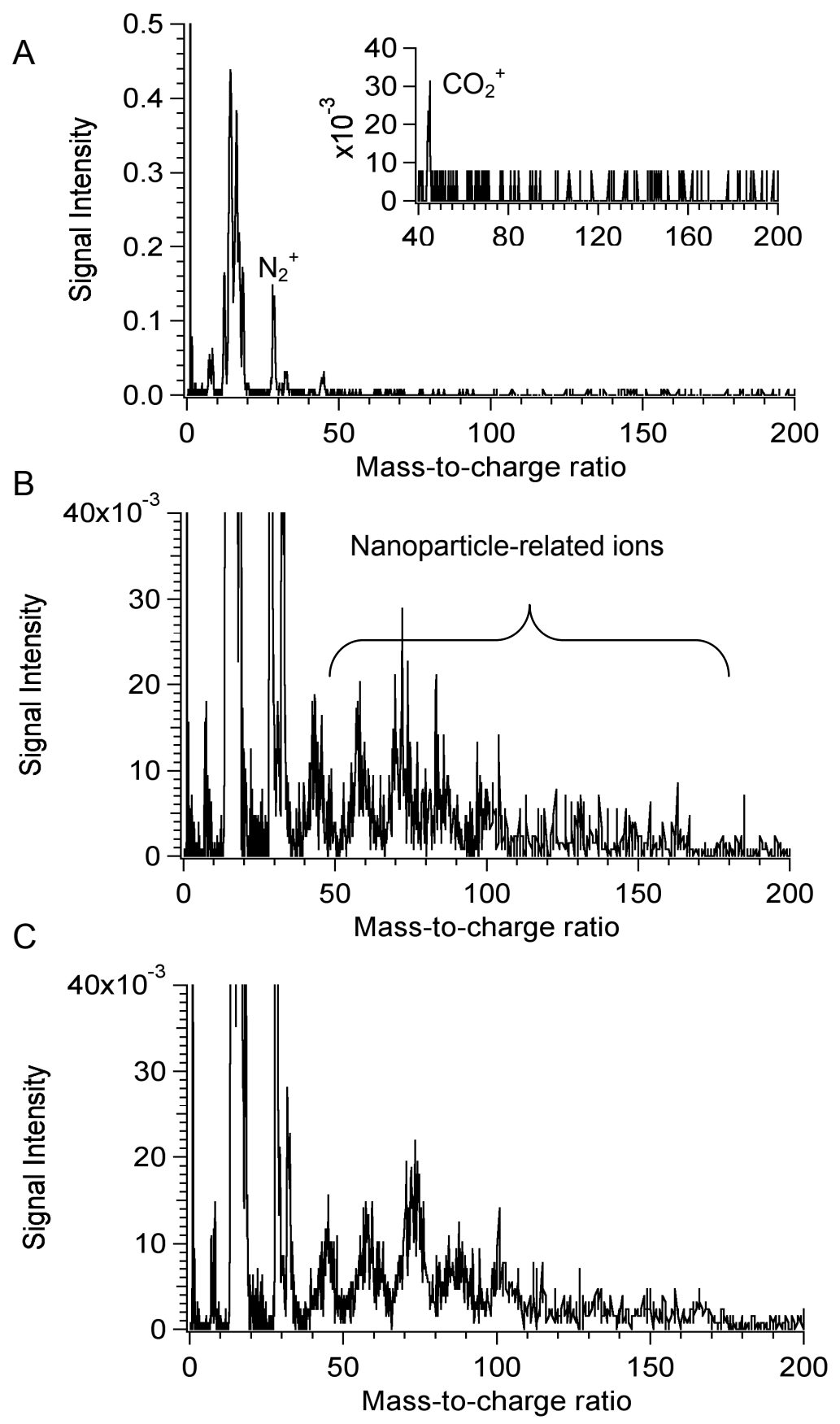

1 Figure 3. Time-of-flight mass spectra of ions created by a single FEL pulse from (A) 2 background gas, (B) the single particle hit in Fig. 4A and (C) the particles hit in Fig. 4B.

4 Mass Spectrometry. Individual particles are intercepted by the FEL randomly, therefore 5 a time-of-flight mass spectrometer (TOFMS) is used to identify particle interception 6 events. Mass spectra can be collected at a rate of $100 \mathrm{kHz}$. Gases delivered through the 7 injector to the interaction region (primarily $\mathrm{N}_{2}, \mathrm{O}_{2}$ and $\mathrm{CO}_{2}$ ) were ionized by the FEL, 
1 resulting in TOFMS detection of the ions $\mathrm{N}^{2+}, \mathrm{N}^{+}, \mathrm{O}^{2+}, \mathrm{O}^{+}, \mathrm{OH}^{+}, \mathrm{H}_{2} \mathrm{O}^{+}, \mathrm{N}_{2}^{+}, \mathrm{O}_{2}^{+}$, and

$2 \mathrm{CO}_{2}{ }^{+}$(Figure 3A). Particle interception events produced ions with mass-to-charge ratios

3 greater than 50 (Figure 3B). These ions are consistent with the ions created from sucrose

4 particles in other high temperature ion sources, such as inductively coupled plasma (7000

$5 \mathrm{~K}) .{ }^{30}$ In this case the particle is heated to $\sim 60000 \mathrm{~K}$ during the interaction ${ }^{5}$ and the

6 mechanism of ion generation has not been elucidated. These are the first mass spectra

7 ever collected from biological materials ionized by a soft X-ray FEL.

9 Single Particle Imaging. X-rays diffracted from material in the interaction region reflect

10 from a graded-period multilayer-coated mirror to a single photon sensitive X-ray CCD

11 while the direct beam passes through a hole in the center of the mirror. For these

12 experiments FLASH was operated in multi-bunch ultrashort pulse mode ${ }^{1}$ to increase the

13 rate of particle interception. Pulse bunches were delivered at $5 \mathrm{~Hz}$ and each bunch

14 consisted of $27 \mathrm{X}$-ray pulses separated by $10 \mu \mathrm{s}$. Each pulse was transform limited and

15 typically dominated by a single mode, i.e. it had almost complete transverse and

16 longitudinal coherence. This high degree of coherence is a requirement for high-

17 resolution imaging and results in the ability to utilize essentially the entire output of the

18 source for our experiments. The mean photon wavelength was $13.5 \mathrm{~nm}$, the average pulse

19 energy was about $20 \mu \mathrm{J}$, equivalent to about $1.4 \times 10^{12}$ photons, and the pulse duration

20 was $15 \pm 5 \mathrm{fs}$.

21 The diffraction pattern recorded by a typical CCD readout when the FEL intercepts a

22 particle is shown in Figure 4A. The strong near vertical and horizontal lines that pass

23 through the center of the pattern are caused by the $200 \mu \mathrm{m}$ square window aperture

24 (Figure 1, (ii)). This aperture is used to block scattered light from the ellipsoidal mirror

25 which would otherwise add noise to the diffraction pattern. We find that a square

26 aperture produces secondary scattering that is localized in the diffraction pattern and

27 which can be easily filtered from the pattern, whereas a circular aperture distributes

28 secondary scattering across the entire pattern. The dark area on the left of the pattern is a

29 shadow due to the mass spectrometer and the vertical line on the right of the pattern is

30 due to a column defect in the CCD. 

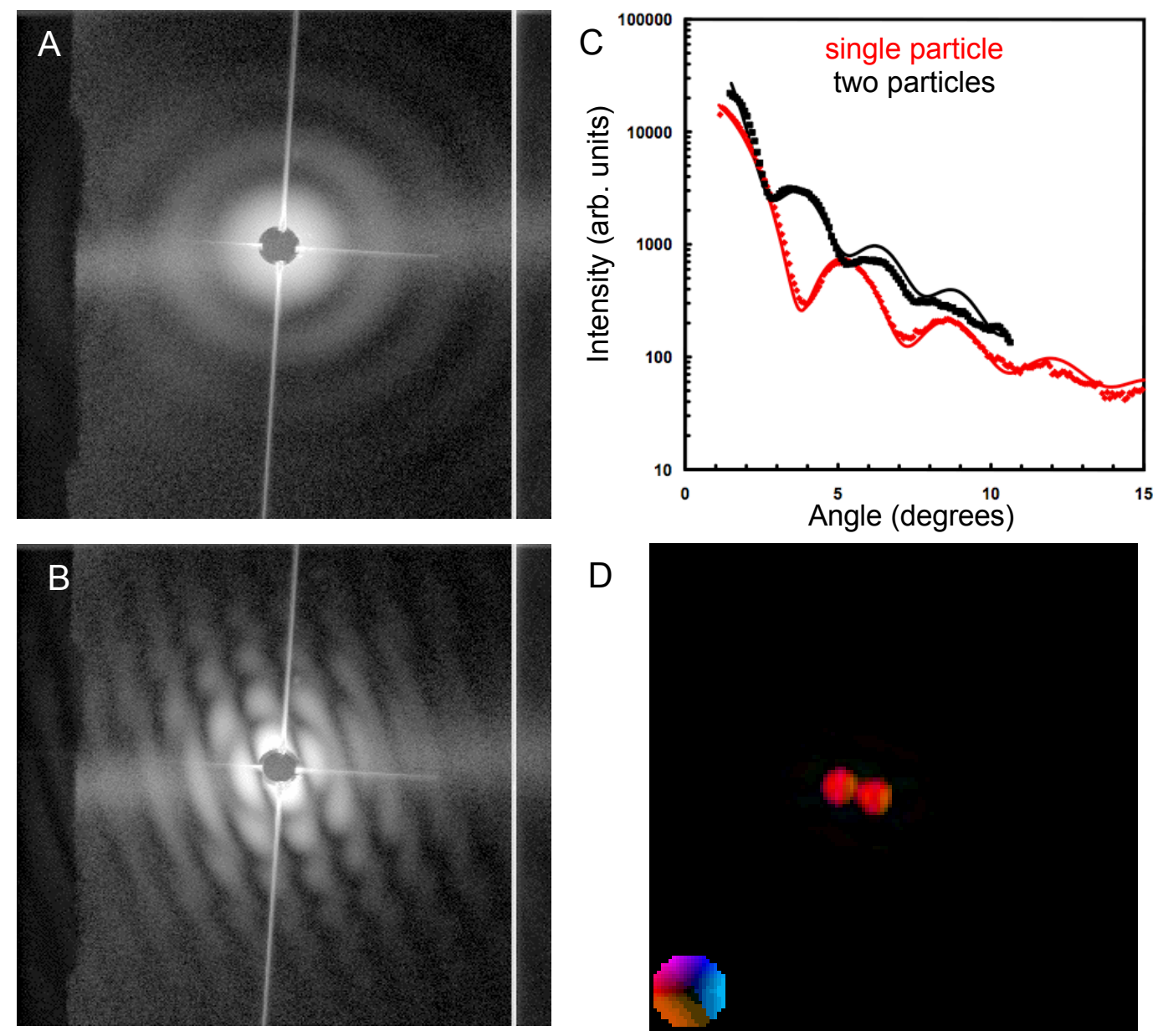

2 Figure 4. FLASH X-ray coherent diffraction patterns of (A) a single particle and (B) two 3 particles. The intensity is shown on a logarithmic grey scale with black denoting 1 4 photon/pixel and white denoting 16500 photons/pixel. Each diffraction pattern is 5 integrated over 243 FEL pulses, one of which intercepted the particles. The entire pattern 6 as detected by the CCD is shown. (C) Radial averages of the angle-resolved signal 7 intensity for the single particle from (A) and the two particles from (B). The markers are 8 experimental data and the solid lines are calculated with Mie theory, which predicts 9 diameters of $248 \pm 10 \mathrm{~nm}$ and $330 \pm 20 \mathrm{~nm}$ (assuming identical size for the pair) 10 respectively. (D) The X-ray image reconstructed from data in (B) shows that the two 11 particles, each $\sim 325 \mathrm{~nm}$ in diameter, are in contact. Pixel size in the reconstruction is 30.3 $12 \mathrm{~nm}$ and the hue denotes the phase of the solution as in the color wheel.

14 This diffraction pattern exhibits the characteristic Airy rings of a spherical particle, 15 indicating that a spherical residue particle is created as the solvent evaporates from the 16 electrospray droplet and the sucrose encapsulates the DNA. The particle diameter $(248 \pm$ 
$1 \quad 10 \mathrm{~nm}$ ) was determined by fitting the radial average of the signal intensity to Mie theory

2 (Figure 4B). The strategy of encasing material in sucrose has the potential to be refined to

3 create molecular tampers predicted to delay the onset of damage to biomolecules during

4 intense single pulse X-ray exposures. ${ }^{31}$

6 Several diffraction patterns indicative of two particles being intercepted by a single FEL

7 pulse simultaneously were also captured unexpectedly (Figure 4C). The diffraction

8 pattern shows fringes due to interference between the scattered waves from two particles.

9 This coherent addition would not be present if two particles are hit by two different

10 pulses. Mass spectrometer data collected for each individual FEL pulse during the CCD

11 acquisition verified that only a single particle interception event occurred, producing the

12 mass spectrum in Figure 3C. The well-defined Airy ring pattern in Figure 4C suggests

13 that the two particles hit by the same pulse are of similar diameter, calculated to be $330 \pm$

$1420 \mathrm{~nm}$ (Figure 4B). The deviation from the Mie theory is attributed to the low signal-to-

15 noise ratio of the diffraction pattern at high angles and the assumption that both particles

16 are of identical diameter. The two minima in the central ring suggest that the distance

17 between the balls in the direction transverse to the beam is the same as their size, i.e. the

18 two particles are in contact and constitute a single dimer particle (see supplemental

19 information). Such a particle could have been created through agglomeration during

20 transport or from two DNA complexes being present in a larger droplet.

21 Real-space images of nanoscale objects can be obtained from single pulse coherent 22 diffraction patterns using iterative projection algorithms for phase retrieval. ${ }^{5,} \mathrm{~A}$ 23 reconstructed image of the two-particle pattern reveals that the two particles were in 24 contact, forming a dimer (Figure 4D). The resolution of this image is $40 \mathrm{~nm}$. Each 25 particle was $325 \mathrm{~nm}$ in diameter, corresponding to the Mie calculated diameter. This is 26 the first image of a substrate-free individual nanoparticle reconstructed from a diffraction 27 pattern created by a single FEL pulse and represents the first step toward imaging of 28 single biomolecules using future X-ray FELs.

30 Single Particle Image Acquisition Rate. Over the length of a 2.5 hour experiment, a 31 total of 105 particle diffraction patterns were recorded. The average rate was 0.012 
1 particle diffraction patterns collected per second or approximately 1 per minute (Figure

$25 \mathrm{~A}$, solid square). The expected rate of image collection for this single particle diffractive

3 imaging technique can be calculated using a modification of an equation derived by Kane

4 et al $^{32,33}$ for determining aerosol mass spectrometry hit rates (HR),

$$
H R=n V T f\left(\frac{r_{L}{ }^{2} h}{r_{P}{ }^{2} v}\right)
$$

6 where $n$ is the aerosol number density, $V$ is the volume flow of aerosol through the inlet,

$7 \quad T$ is the inlet transmission efficiency, $h$ is the X-ray laser beam height (width along the 8 particle beam axis), $v$ is the particle velocity and $r_{L}$ and $r_{P}$ are the radius of the FEL pulse 9 and the particle beam, respectively. The fixed repetition rate, $f$, of FLASH and the 10 random arrival of particles transmitted through the aerodynamic lens results in hits 11 occurring only when there is coincidental overlap of individual particles with the pulsed 12 laser.

14 Encapsulated DNA nanoparticles were prepared as an aerosol with a concentration of $n$ $15=3 \times 10^{4}$ particles $/ \mathrm{cm}^{3}$, were sampled by the aerodynamic lens inlet at a rate of $V=14.33$ $16 \mathrm{~cm}^{3} / \mathrm{second}$ and traveled through the interaction region at $v=150 \mathrm{~m} / \mathrm{s}$. FEL pulses were 17 delivered at $f=135$ pulses/second. The interaction volume (Figure 1, point (iii)) is 18 defined by the particle beam diameter $\left(2 r_{P}=250 \mu \mathrm{m}\right)$ and the FEL focus $\left(2 r_{L}=20 \mu \mathrm{m}\right.$ in 19 diameter). Using these parameters Eqn. (1) predicts a hit rate of 0.06 particles/second, 20 assuming a transmission efficiency of $T=10$ percent and setting $h$ equal to the particle 21 beam diameter (Figure 5A). The average hit rate ( 0.07 particles/second) over a 200 22 second period of our experiment agreed with the predicted hit rate (Figure 5B). The 23 discrepancy between the predicted and measured value over the longer experiment is in 24 part due to steering of the particle beam and down time when changing between pure 25 sucrose and sucrose/DNA samples. We note that the hit rate increases linearly with laser 26 repetition rate, so without modification of our experimental apparatus the hit rate will 27 improve as the FEL pulse rate increases: FLASH has recently been operated at 700 28 pulses/second with design specifications of over 1500 pulses/second, ${ }^{2}$ giving an 29 immediate factor of between 5 to 11 increase in hit rate. 

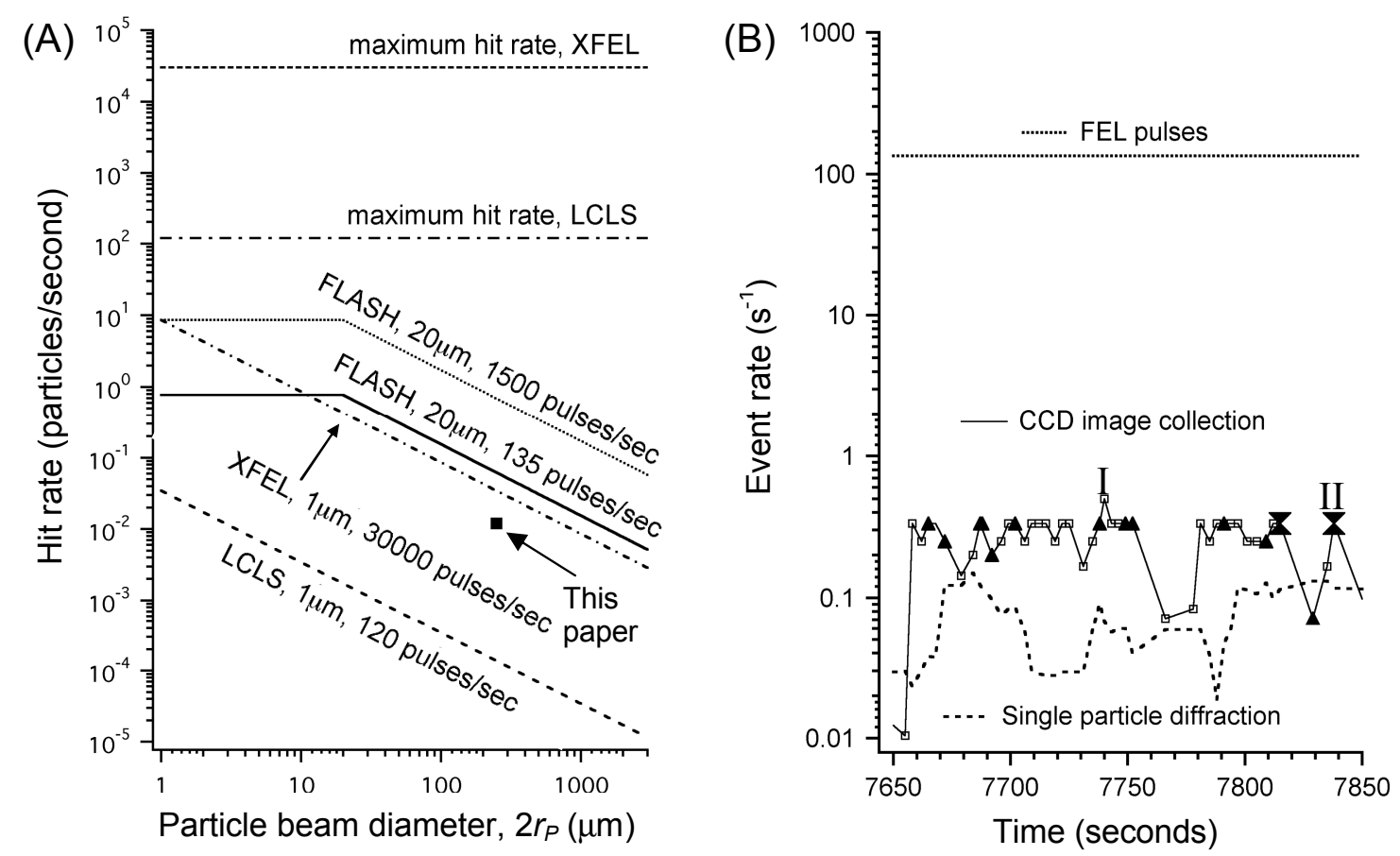

3 Figure 5. (A) Predicted hit rates at FLASH, XFEL and LCLS of nanoparticles, 4 electrosprayed to an aerosol concentration of $3 \times 10^{4}$ particles $/ \mathrm{cm}^{3}$, for varying particle 5 beam diameter. The maximum hit rate is equal to the maximum number of pulses per 6 second from the FEL. Annotation denotes X-ray source, FEL beam diameter, and number 7 of pulses delivered per second. The filled square is the average hit rate measured at 8 FLASH during a 2.5 hour experiment with 135 FEL pulses/second. (B) Comparison of 9 the rates of FEL pulse (and mass spectrometer acquisition), CCD image acquisition and 10 single particle diffraction events over a 200 second period of a single particle diffractive 11 imaging experiment. Markers on CCD line: open square $=$ no particle hit, triangle = 12 single particle hit, double triangle $=$ dimer particle hit. Diffraction patterns collected at 13 CCD readouts I, and II are shown in Figure 4.

Discussion. Experiments at future hard-X-ray FELs, such as the Linac Coherent Light 16 Source (LCLS) and the European X-ray Laser (XFEL), are predicted to enable the 17 determination of three-dimensional biomolecular structure at near-atomic resolution 18 without crystallization. Such an experiment is currently predicted to require $>10^{4}$ images 19 of identical biomolecules, ${ }^{8,9}$ thus maximizing the hit rate achievable by single particle 20 imaging is critical to reduce the data acquisition time. Further exacerbating the challenge 
1 is that the X-ray beam must be focused down to much smaller spot sizes, typically below

$21 \mu \mathrm{m}$, to increase the incident intensity and ensure adequate signal at the higher photon

3 momentum transfers (higher resolutions) that the shorter wavelength affords. This will

4 immediately decrease the efficiency of the apparatus demonstrated here by a factor of

5400 or more based on the $20 \mu \mathrm{m}$ diameter FEL beam at FLASH.

FEL laser repetition rate is another critical factor. At the European XFEL the inefficiencies due to FEL beam size will be made up by the increased FEL pulse rate of up to 30,000 pulses $/$ second. For a source aerosol concentration of $3 \times 10^{4}$ particles $/ \mathrm{cm}^{3}$,

10 this results in an expected hit rate that is only half of that measured at FLASH during the

11 experiments reported in this letter. Hit rates at LCLS may be significantly lower because

12 the planned pulse rate is 120 pulses/second. We summarize the potential hit rates at

13 FLASH and future FEL facilities in Fig. 5A, considering only FEL machine parameters

14 and assuming no further improvements in aerodynamic focusing or aerosol density. This

15 is therefore a pessimistic estimate assuming no further sample handling development.

17 Image acquisition rates will be improved in a number of ways independent of the X-ray 18 source used. For example, improved particle beam focusing to match the sub-micron 19 FEL beam size may be possible through developments in the aerodynamic lens stack

20 itself. If the exit nozzle of the aerodynamic lens stack is positioned in closer proximity to 21 the interaction region, the effect of the divergence angle (currently 0.5 to 3 degrees) of

22 the particle beam imparted by gas expansion will be reduced. It may also be possible to 23 increase the particle density sampled by the aerosol handling system using multi-nozzle 24 electrospray or droplet sources. ${ }^{34,35}$ imaging is that it is a leak in the vacuum chamber that continuously samples from atmospheric pressure (at a rate of $14.33 \mathrm{~cm}^{3} \mathrm{~s}^{-1}$ for the current design). Thus any aerosol

29 existing near the inlet is passively sampled. This enables us to utilize the advantages of 30 well-characterized atmospheric aerosol sources such as electrospray for preparing 31 samples to be imaged. Many other atmospheric pressure aerosolization methods, 
1 including flames, atomizers, and lasers, can also be used. All of these aerosol sources can

2 be interchanged instantaneously or operated simultaneously. Many of these aerosol

3 sources can generate particle concentrations equivalent to or greater than the $3 \times 10^{4}$

4 particles $/ \mathrm{cm}^{3}$ used here. Each type of source generates particles and size distributions

5 with unique characteristics that make them more or less applicable to the nanoscale

6 biomaterials we studied by electrospray. For example, aerosol sources used in atomic

7 cluster research often operate at concentrations of $10^{10}$ clusters $/ \mathrm{cm}^{3}$ and will certainly be

8 a useful source for single particle X-ray diffractive imaging of core-shell structured

9 atomic clusters. However, they are currently not suitable for biomolecule imaging. To put

10 these aerosol concentrations into a real-world perspective, measurements of the aerosol

11 concentration generated by automobiles near highways show a total of $10^{4}-10^{6}$

12 particles $/ \mathrm{cm}^{3},{ }^{36}$ suggesting it may even be possible to image environmental particles.

14 Another advantage of aerodynamic lens interface is its ability to continuously operate 15 for many hours without clogging, a crucial characteristic for successful utilization of 16 precious X-ray FEL beam time. The key limitation of this method is the current inability 17 to time arrival of particles with the FEL pulses. Single particle sources designed to 18 deliver proteins in synchronization with the X-ray pulses are also being developed ${ }^{37}$ and, 19 if successful, may overcome the inefficiency of operation in free-fire mode. It is 20 anticipated that continued development of single particle sources of all types will increase 21 the number of accessible sample types and raise the achievable hit rate to the maximum 22 levels available for both LCLS and XFEL. Detectors designed to accommodate single 23 particle diffraction data acquisition at such high rates are in development.

25 The ultimate goal for single molecule X-ray imaging is the delivery of a sufficient 26 number of identical molecules to enable 3D reconstruction. Any diffraction patterns of 27 non-identical molecules collected will reduce the effective hit rate and increase the 28 complexity of classifying images of molecules in different orientations. Future 29 experiments will make use of particle size selection technology such as differential 30 mobility methods. ${ }^{20}$ This approach can be used to remove contaminants such as residue 31 particles or to select molecular conformations, potentially reducing the complexity of 
1 classifying diffraction patterns. Also note that the thickness of the sucrose coatings,

2 which in our experiments had a broad distribution, can currently be controlled to less than

$31 \mathrm{~nm}^{29}$

5 The resolution obtained in single pulse X-ray imaging experiments is limited in part by 6 the onset of damage in the sample due to the intense X-ray pulse. Depending on the pulse 7 duration, the resolution limit may be $0.5 \mathrm{~nm}$ for hard-X-ray FEL pulses, or even less with 8 encapsulated particles. ${ }^{31}$ For the pulse fluences and wavelength of these experiments, 9 calculations show that the diffraction pattern formed by the pulse would be essentially the 10 same as the undamaged case and that any expansion of the particle would certainly be 11 much less than the resolution length of $40 \mathrm{~nm}^{38}$ Furthermore, diffractive imaging of well 12 characterized nano-structured objects at FLASH, at 32-nm wavelength, have shown no 13 measurable damage. ${ }^{5}$ In the experiments reported here we are not able to probe the 14 extent of damage in the sample due to unknown shape and size characteristics of the 15 individual sucrose-encapsulated DNA particles imaged during a specific pulse. Future 16 work investigating the onset of damage in particles in free flight will utilize size17 monodisperse spherical nanoparticles that have been diameter-selected by differential 18 mobility methods ${ }^{20}$ and sampled directly into the aerodynamic lens stack. This approach 19 will be an extension of our method for measuring X-ray pulse-induced damage at 20 femtosecond time resolutions with size-selected spherical nanoparticles deposited onto 21 silicon nitride membranes. ${ }^{4}$

23 Our results unequivocally show the feasibility of single-particle X-ray diffractive 24 imaging using FELs. It is expected that future X-ray FEL facilities will enable nanometer 25 to atomic scale resolution imaging of non-crystalline nanoscale materials, such as large 26 biomolecules, viruses or cells, inorganic particles and environmental particles. Single 27 particle X-ray diffractive imaging will provide new capabilities for following the 28 dynamics of materials at the atomic time and length scales, potentially resulting in

29 significant advances in nanotechnology development and structural biology. 
1 ACKNOWLEDGEMENTS: Special thanks are due to the scientific and technical staff of 2 the FLASH at DESY, Hamburg, in particular to R. Treusch, J. Schneider, S. Dusterer, T. 3 Tschentscher, J. Feldhaus, R.L. Johnson, U. Hahn, T. Nuñez, K. Tiedtke, S. Toleikis,

4 E.L. Saldin, E.A. Schneidmiller, and M.V. Yurkov. We are grateful to our collaborators

5 in T. Möller's group at Technische Universitat Berlin for accomodating our experiment

6 in their vacuum chamber. We also thank D. Shapiro, T. McCarville, F. Weber, and M.

7 Haro for technical help with these experiments. This work was supported by the

8 following agencies: The U.S. Department of Energy by Lawrence Livermore National

9 Laboratory in part under Contract W-7405-Eng-48 and in part under Contract DE-AC52-

10 07NA27344, Lawrence Livermore National Laboratory (the project 05-SI-003 from the

11 Laboratory Directed Research and Development Program of LLNL); The National

12 Science Foundation Center for Biophotonics, University of California, Davis, under

13 Cooperative Agreement PHY 0120999; The National Center for Electron Microscopy

14 and the Advanced Light Source, Lawrence Berkeley Lab, under DOE Contract DE-

15 AC02-05CH11231; the DOE Office of Science to the Stanford Linear Accelerator

16 Center; the European Union (TUIXS); The Swedish Research Council; The Swedish

17 Foundation for International Cooperation in Research and Higher Education; The

18 Swedish Foundation for Strategic Research; The Sven and Lilly Lawskis Fund (Doctoral

19 Fellowship to MMS) and the Natural Sciences and Engineering Research Council of

20 Canada (NSERC Postdoctoral Fellowship to MJB). UCRL-JRNL-235405

\section{REFERENCES:}

1. Ayvazyan, V.; Baboi, N.; Bahr, J.; Balandin, V.; Beutner, B., et al. Eur. J. Phys. D 2006, 37, 297-303.

2. Ackermann W.; Asova G.; Ayvazyan V.; Azima A.; Baboi N., et al. Nat. Photon. 2007, 1, (6), 336-342.

3. Gaffney, K. J.; Chapman, H. N. Science 2007, 316, (5830), 1444-1448.

4. Chapman, H. N.; Hau-Riege, S. P.; Bogan, M. J.; Bajt, S.; Barty, A., et al. Nature 2007, 448, (9), 676-679.

5. Chapman, H. N.; Barty, A.; Bogan, M. J.; Boutet, S.; Frank, M., et al. Nat. Phys. 2006, 2, (12), 839-843.

6. Fienup, J. R. Appl. Opt. 1982, 21, 2758-2769.

7. Marchesini, S. Rev. Sci. Instr. 2007, 78, (4), 011301.

8. Neutze, R.; Wouts, R.; van der Spoel, D.; Weckert, E.; Hajdu, J. Nature 2000, 406, 752-757. 
9. Huldt, G.; Szoke, A.; Hajdu, J. J. Struct. Biol. 2003, 144, 219-227.

10. Spence, J. C. H.; Doak, B. Phys. Rev. Lett. 2004, 92, 198102.

11. Piseri, P.; Tafreshi, H. V.; Milani, P. Curr. Op. Solid State Mater. Sci. 2004, 8, (3-4), 195-202.

12. Carbone, R.; Giorgetti, L.; Zanardi, A.; Marangi, I.; Chierici, E., et al. Biomaterials 2007, 28, (13), 2244-2253.

13. Noble, C. A.; Prather, K. A. Mass Spectrom. Rev. 2000, 19, 248-274.

14. Wilson, K. R.; Peterka, D. S.; Jimenez-Cruz, M.; Leone, S. R.; Ahmed, M. Phys. Chem. Chem. Phys. 2006, 8, (16), 1884-1890.

15. Shu, J.; Wilson, K.; Ahmed, M.; Leone, S. R. J. Chem. Phys. 2006, 124, 034707.

16. Liu, P.; Ziemann, P. J.; Kittleson, D. B.; McMurry, P. H. Aerosol Sci. Tech. 1995, 22, 314-324.

17. Liu, P.; Ziemann, P. J.; Kittleson, D. B.; McMurry, P. H. Aerosol Sci. Tech. 1995, 22, 293-313.

18. Wexler, A. S.; Johnston, M. V., Real-time single-particle analysis. In Aerosol Measurement, Baron, P. A.; Willeke, K., Eds. John Wiley \& Sons: Hoboken, New Jersey, 2005; pp 365-386.

19. Chen, D. R.; Pui, D. Y. H.; Kaufman, S. L. J. Aerosol Sci. 1995, 26, (6), 963-977.

20. Bogan, M. J.; Benner, W. H.; Hau-Riege, S. P.; Chapman, H. N.; Frank, M. J. Aerosol Sci. 2007, 38, (11), 1119-1128.

21. O'Shea, J. N.; Taylor, J. B.; Swarbrick, J. C.; Magnano, G.; Mayor, L. C., et al. Nanotechnology 2007, 18, (3), 035707.

22. Bacher, G.; Szymanski, W. W.; Kaufman, S. L.; Zollner, P.; Blaas, D., et al. J. Mass Spectrom. 2001, 36, 1038-1052.

23. Hogan, C. J. J.; Kettleson, E. M.; Ramaswami, B.; Chen, D. R.; Biswas, P. Anal. Chem. 2006, 78, (3), 844-852.

24. Bothner, B.; Siuzdak, G. ChemBioChem 2004, 5, 258-260.

25. Tito, M. A.; Tars, K.; Valegard, K.; Hajdu, J.; Robinson, C. V. J. Amer. Chem. Soc. 2000, 122, (14), 3550-3551.

26. Blades, A. T.; Ikonomou, M. G.; Kebarle, P. Anal. Chem 1991, 63, (19), 2109-2114.

27. Rothemund, P. W. K. Nature 2006, 440, 297-302.

28. Hau-Riege, S. P.; Szoke, H.; Chapman, H. N.; Szoke, A.; Marchesini, S., et al. Acta Crystallographica Section A 2004, 60, (4), 294-305.

29. Kaufman, S. L. Anal. Chim. Acta 2000, 406, (1), 3-10.

30. Taylor, V. F.; March, R. E.; Longerich, H. P.; Stadey, C. J. Int. J. Mass Spectrom. 2005, 243, (1), 71-84.

31. Hau-Riege, S. P.; London, R. A.; Chapman, H. N.; Szoke, A.; Timneanu, N. Phys. Rev. Lett. 2007, 98, 198302.

32. Kane, D. B.; Johnston, M. V. Environ. Sci. Technol. 2000, 34, 4887-4893.

33. Kane, D. B.; Oktem, B.; Johnston, M. V. Aerosol Sci. Tech. 2001, 34, (6), 520 - 527.

34. Deng, W.; Klemic, J. F.; Li, X.; Reed, M. A.; Gomez, A. J. Aerosol Sci. 2006, 37, 696-714.

35. Bocanegra, R.; Galan, D.; Marquez, M.; Loscertales, I. G.; Barrero, A. J. Aerosol Sci. 2005, 36, 1387-1399.

36. Kittelson, D. B.; Watts, W. F.; Johnson, J. P.; Remerowki, M. L.; Ische, E. E., et al. Inhalation Toxicology 2004, 16, (11 supp 1), 31 - 39. 
1 37. Weierstall, U.; Doak, R. B.; Spence, J. C. H.; Starodub, D.; Shapiro, D., et al. Exp. 2 Fluids 2007, in press.

3 38. Hau-Riege, S. P.; London, R. A.; Bergh, M.; Chapman, H. N. Phys. Rev. E 2007, 76, 4046403. 